Research Article

\title{
Modeling and Stochastic Model Updating of Bolt-Jointed Structure
}

\author{
Ming Zhan $\mathbb{D}^{1},{ }^{1}$ Qintao Guo $\mathbb{D}^{1},{ }^{1}$ Lin Yue, ${ }^{1}$ and Baoqiang Zhang $^{2}$ \\ ${ }^{1}$ Nanjing University of Aeronautics and Astronautics, Nanjing, China \\ ${ }^{2}$ Xiamen University, Xiamen, China \\ Correspondence should be addressed to Qintao Guo; guo_qintao@nuaa.edu.cn
}

Received 12 August 2018; Revised 30 September 2018; Accepted 10 October 2018; Published 6 November 2018

Academic Editor: Marco Tarabini

Copyright (c) 2018 Ming Zhan et al. This is an open access article distributed under the Creative Commons Attribution License, which permits unrestricted use, distribution, and reproduction in any medium, provided the original work is properly cited.

Bolt-jointed structure is widely used in engineering fields. The dynamic characteristics of bolt-jointed structure are complex, and there is a variety of uncertainties in the jointed structure. In this study, modeling and updating of a typical bolt-jointed structure are investigated. In modeling terms, three-dimensional brick elements are used to represent the substructures, and thin-layer elements with virtual material properties are employed to represent the joint interface. Modal tests and experimental modal analysis of substructures and built-up structure are performed. A hierarchical model updating strategy based on Bayesian inference is applied to identify the unknown parameters in the substructures model and those in the overall model. Radial basis function (RBF) models are used as surrogates of time-consuming finite element model with high resolution to avoid the enormous computational cost. The results indicate that the updated model can reproduce modal frequencies used in updating and can predict those not used in the updating procedure.

\section{Introduction}

Modeling and simulation (M\&S) is extensively used in the engineering community to represent actual structures, and it is the third pillar of science [1]. A reasonable finite element model (FEM) makes a variety of model-based activities, such as response analysis and prediction, structural health monitoring, and damage identification, possible. As a result, a precise FEM is of great significance to characterize the structural behavior. In the reality, engineering structures are composed of several relatively simple substructures that are connected together by bolt joints which results in the overall structure to be a complex system.

The precision of outputs obtained from FEM is greatly dependent on the model form and model parameters what makes the finite element modeling become a challenging task. In the bolt-jointed structure, the modeling of substructures and joint interfaces are the two main aspects that affect the model performance. As a rule, substructures can be modeled by conventional elements, such as shell and brick elements, or in some other multiscale ways. However, joint interfaces are usually represented by some equivalent elements, including but not limited to node-to-node elements, zero-thickness elements, and thin-layer elements as well as refined elements with high fidelity. Bograd et al. gave a summary of different approaches for modeling the dynamics of mechanical joints in composition structures [2]. Sandia National Laboratories released a handbook on the dynamics of jointed structures which includes modeling, experiments, and suggestions for future works [3]. Mayer and Gaul used segment-to-segment contact elements to define a contact interface in an assembled structure [4]. Luan et al. proposed a bilinear spring dynamical model and used it to analyze a pipe structure with bolted flange joints [5]. Zhao et al. employed the fractal theory to obtain the stiffness and damping of the contact interface in a bolted assembly [6]. Li et al. applied the six-parameter Iwan model to describe the nonlinear mechanisms of bolt joints [7]. Wang et al. investigated the equivalent parameters of mechanical characteristics of bolt joints in a complex mechanical system by thin-layer elements [8]. Xiang et al. 
proposed an improved spring method and used it in the modeling of the composite bolt-jointed structures [9].

However, the parameters in the incipient FEM built on the basis of the designed blue print are usually determined according to design handbooks or analyst's experience. Apparently, responses, calculated utilizing such a model, often differ from those measured from a real-world structure. Model updating is a powerful approach to minimize the difference between the model analysis results and the corresponding measurements. Mottershead and Friswell gave a summary of the model updating on the structural dynamics and published a treatise of this field $[10,11]$. In the last two decades, a variety of works on modeling and updating of bolt joints have been put forward and implemented. Ahmadian et al. carried out model updating of joints in the AWE-MACE system utilizing a sensitivity method within the linear range [12]. Zhai et al. constructed a parametric model of bolt joints of aeroengine stator system and updated the model with multicharacteristic responses [13]. Cunha et al. applied the model updating method to identify the stiffness of bolt joints in civil construction structural systems [14]. Adel et al. presented a simple and applicable model to predict the dynamic behavior of bolt joints and updated the model using modal test data [15]. An in-depth understanding of model updating in structural dynamics can be acquired from Reference [16].

In the early stage, model updating was performed under the deterministic framework; that is, model parameters were estimated utilizing a single set of measurement. However, uncertainties are irremissible in both model form and model parameters. These uncertainties are usually introduced by simplification of geometric features, assumption of constitutive models, and representation of joint interfaces. Ibrahima and Pettit presented an overview on the nonlinear characteristics and uncertainties in bolt joints pertaining to their dynamics [17]. For the concern of multisource uncertainties in various aspects of structure dynamics, the stochastic model updating which refers to determine the range or probability density function of model parameters utilizing several experimental tests was put forward and promoted in recent years. A variety of methods were proposed for stochastic model updating, including the perturbation method, interval analysis, Monte Carlo simulation, fuzzy arithmetic, and Bayesian inference. Khodaparast et al. developed the model updating equations using the sensitivity method and estimated the statistical moments of updating parameters utilizing perturbation methods [18]. Jiang et al. identified the statistical characteristics of model parameters using vibration test data and first-order perturbation method [19]. Khodaparast et al. investigated the Kriging predictor metamodel and presented an interval model updating approach based on the Kriging predictor [20]. Mares et al. explained the theory of stochastic model updating using the Monte Carlo simulation and applied the theory to a benchmark structure [21]. Haag et al. presented a method for the validation of models based on the inverse fuzzy arithmetic [22]. Wan and Ren. combined the Bayesian inference and Gaussian process model in the stochastic model updating and illustrated the proposed framework utilizing a simulated plate and a real-world bridge [23]. Goller et al. elaborated the fundamental rule of Bayesian model updating and validated the method utilizing numerical examples [24].

However, in the most stochastic model updating activities, iterative computations or huge amount of sampling simulations are extremely time-consuming, especially for complex FEMs with high fidelity. For the sake of improving computational efficiency, surrogate models and component mode synthesis methods are investigated in the issues of structural dynamics. Rui et al. developed a stochastic model updating method by combining the stochastic response surface method with the Monte Carlo inverse error propagation [25]. Fang et al. proposed a simple and cost-efficient method for stochastic model updating utilizing the response surface method and Monte Carlo simulation [26]. Dohnal et al. addressed the joint uncertainties in a finite element model based on component mode synthesis and the stochastic reduced basis method [27]. Yin et al. detected a defect in a structure with bolt joints using the model reduction method and system mode concept under the Bayesian framework [28].

The remainder of this manuscript is organized as follows: in Section 2, the basic theory of stochastic model updating based on the Bayesian inference, surrogate modeling and evaluation, basic theory of thin-layer elements are briefly introduced. Section 3 is devoted to the real bolt lapped structure, including finite element modeling, surrogate modeling, and model updating of substructures and the overall structure. The work ends with conclusions in Section 4.

\section{Methodology}

Modeling and simulation of bolt joints has been an active research subject in mechanical engineering and some other engineering fields. Model updating is a synthesis approach that combines numerical simulations and experimental tests. With the popularization of model updating methodologies and awareness of uncertainties, stochastic model updating has been widely used. Details about methodologies used in this article will be introduced in the following parts.

2.1. Stochastic Model Updating Based on Bayesian Inference. Stochastic model updating based on the Bayesian inference is a process to evaluate the probability distribution functions (PDFs) by combining the existing knowledge about updating parameters with experimental observations [23]. In essence, the Bayesian inference approach updates the prior PDFs of updating parameters utilizing experimental data and resulting in posterior PDFs.

Given that $\boldsymbol{\theta}$ is the vector of updating parameters and $\mathbf{D}$ is the information matrix of experimental observations, the updated PDFs of updating parameters are governed by the Bayesian inference theorem:

$$
p(\boldsymbol{\theta} \mid \mathbf{D})=\frac{p(\mathbf{D} \mid \boldsymbol{\theta}) p(\boldsymbol{\theta})}{p(\mathbf{D})},
$$


where $p(\boldsymbol{\theta} \mid \mathbf{D})$ is the posterior PDFs of updating parameters given for the experimental observations, $p(\mathbf{D} \mid \boldsymbol{\theta})$ is the likelihood function, $p(\boldsymbol{\theta})$ is the prior PDFs of updating parameters, and $p(\mathbf{D})$ is the marginal PDF of experimental observations.

The prior PDFs, $p(\boldsymbol{\theta})$, represents the understanding of updating parameters before model updating, and it is defined based on analyst's experience, expert's judgment, design specification, or some previous knowledge of similar conditions. The posterior $\operatorname{PDF} p(\boldsymbol{\theta} \mid \mathbf{D})$ is the result of model updating by combining prior information and experimental measurements, and it can be considered as the updated distribution of selected updating parameters.

In formula (1), the denominator, $p(\boldsymbol{\theta} \mid \mathbf{D})$, is a normalized constant given by $p(\mathbf{D})=\int p(\mathbf{D} \mid \boldsymbol{\theta}) p(\boldsymbol{\theta}) d \boldsymbol{\theta}$, which ensures that $\int p(\boldsymbol{\theta} \mid \mathbf{D}) d \boldsymbol{\theta}=1$. So that, ignoring the normalized denominator yields $p(\boldsymbol{\theta} \mid \mathbf{D}) \propto p(\mathbf{D} \mid \boldsymbol{\theta}) p(\boldsymbol{\theta})$, and $\propto$ is a symbol that stands for being proportional.

The likelihood function $p(\boldsymbol{\theta} \mid \mathbf{D})$ represents a similar degree of observations between experiment and simulations, and it is given by

$$
p(\mathbf{D} \mid \boldsymbol{\theta})=\prod_{i=1}^{n} \frac{1}{\sigma \sqrt{2 \pi}} \exp \left(-\frac{(y(\boldsymbol{\theta})-\bar{y})^{2}}{2 \sigma^{2}}\right),
$$

where $y(\boldsymbol{\theta})$ is model predictions, $\bar{y}$ is the mean vector, and $\sigma$ is the covariance matrix of experimental measurements, respectively.

Actually, models of engineering structures are usually complex and unlikely to be solved analytically. As a result, the obtainment of the posterior PDFs of updating parameters utilizing direct multidimensional integration is impractical. Markov chain Monte Carlo (MCMC) sampling is a promising approach to generate sampling points according to a specific rule for rejection, and it can avoid multidimensional integration in the Bayesian inference effectively. In essence, the MCMC sampling is a dynamical Monte Carlo simulation in which the distribution of sampling points varies with the process of simulation. The success of MCMC sampling is greatly dependent on the degree of proximity between the proposal PDFs of updating parameters with the target ones. The Metropolis-Hastings $(\mathrm{MH})$ sampling algorithm is one of the most widely used MCMC sampling methods. However, the efficiency of $\mathrm{MH}$ sampling algorithm is affected by model dimensions. For a model with low dimensions, a quick and perfect convergence can be achieved, but the result is not contrary for a higher dimensional model. For the problem of model updating presented in this article, a revised $\mathrm{MH}$ algorithm called as the Delayed Rejection Adaptive Metropolis-Hastings (DRAM) sampling algorithm is proposed [29]. The DRAM is a combination of delayed rejection method and adaptive $\mathrm{MH}$ algorithm. The main advantages of DRAM algorithm are as follows: (1) adaptive $\mathrm{MH}$ algorithm uses the ergodic information of a chain to adjust the proposal distribution, and it is a global strategy and (2) delayed rejection increases the efficiency of sampling based on the rejected proposal only within each time step, which means that it is a local strategy.
2.2. Surrogate Modeling and Evaluation. The engineering community demonstrated a growing interest in applying surrogate models to replace a complex FEM for practical engineering problems. Surrogate modeling is a synthesis technology of experimental design and approximation method. Experimental design generates a set of design variables and calculates model responses utilizing the FEM. Approximation method builds the relationship between model responses and design variables. A surrogate model with high precision used in engineering problems can avoid a huge amount of computation effectively.

The common experimental design methods are orthogonal design, uniform design, and Latin hypercube sampling (LHS). However, the selection of experimental design method should be cautious because a set of inappropriate sampling points can bring about low accuracy in the surrogate models and even to a worse result with the failure to construct these models [30]. LHS is a stratified sampling process that can reflect the distribution of variables over the whole design space. Hence, the LHS method is employed in this paper to generate sampling points for the construction of surrogate models.

The approximation methods including but not limited to the polynomial response surface method (PRSM) [26], Kriging [20], support vector machine (SVM) [31], Gaussian process (GP) model [32], and radial basis function (RBF) model [26] are commonly used. A RBF model is constructed based on the basis function, which is symmetric and centered at each sampling point. A brief depiction of RBF model is given as follows:

$$
f(\mathbf{x})=\sum_{i=1}^{N_{s}} \lambda_{i} \phi\left(\mathbf{d}_{i}\right)
$$

where $f(\mathbf{x})$ is the approximation of objective function, $N_{s}$ is the total number of sampling points, $\lambda_{i}$ is the weight coefficient which is to be determined by the process of approximation, and $\phi\left(\mathbf{d}_{i}\right)$ is the basis function of Euclidean distance $\mathbf{d}_{i}$.

The Euclidean distance $\mathbf{d}_{i}=\left\|\mathbf{x}-\mathbf{x}_{i}\right\|$ is a normal value, which represents the distance from the design variables $\mathbf{x}$ to the normalized design variables $\mathbf{x}_{i}$ at the $i$ th training point. Different forms of basis function used in constructing a RBF model are listed in Table 1.

The symbol $c$ in the mathematical expression of different basis function, named as the smoothing coefficient, is a vital factor that determines the precision of RBF model.

The precondition of using a surrogate model to replace the FEM is that the surrogate model shall have sufficient precision over the design space. Therefore, the accuracy evaluation of surrogate model is necessary after it is constructed. In addition, the accuracy evaluation of surrogate model at points used in constructing the model and those resampled from the design space should be conducted simultaneously. Two assessment criteria, known as coefficient of determination and root mean square error, are employed in this paper [33]: 
TABLE 1: Different forms of basis function for RBF model.

\begin{tabular}{lc}
\hline Basis function & Mathematical expression \\
\hline Gaussian & $\phi(d)=e^{-c d^{2}}, 0<c \leq 1$ \\
Multiquadric & $\phi(d)=\sqrt{d^{2}+c^{2}}, 0<c \leq 5$ \\
Inverse quadric & $\phi(d)=1 / d^{2}+c^{2}, 0<c \leq 5$ \\
Inverse multiquadric & $\phi(d)=1 / \sqrt{d^{2}+c^{2}}, 0<c \leq 5$ \\
\hline
\end{tabular}

$$
\begin{gathered}
R^{2}=1-\frac{\sum_{j=1}^{N_{t}}\left(Y_{j}(x)-Y_{R j}\right)^{2}}{\sum_{j=1}^{N_{t}}\left(Y_{R j}-\overline{Y_{R J}}\right)^{2}}, \\
\text { RMSE }=\frac{1}{\overline{Y_{R}}} \sqrt{\frac{1}{N_{t}} \sum_{j=1}^{N_{t}}\left(Y_{j}(x)-Y_{R J}\right)^{2}},
\end{gathered}
$$

where $N_{t}$ is the number of points used in the accuracy evaluation, $Y_{j}(x)$ is the $j$ th value calculated from the surrogate model, $Y_{R j}$ is the corresponding value obtained from FEM, and $\overline{Y_{R J}}$ is the mean value of sampling values. The closer the $R^{2}$ is to 1 and the closer the RMSE is to 0 , the more accurate the surrogate model is.

2.3. Basic Theory of Thin-Layer Element. Modeling bolt joints utilizing thin-layer elements is an effective way to investigate dynamical characteristics of this kind of structures. The idea of thin-layer element was first proposed by Desai, and then thin-layer elements were applied to model the soil-structure interface as well as rock joints $[4,34]$. The thin-layer element is an isoparametric brick element with very small but finite thickness. A brick element with length $l$, width $w$, and thickness $t$, as shown in Figure 1, is taken as the example to elaborate the formulation of thin-layer element. The virtual work of a thin-layer element can be expressed as

$$
\delta W=\iiint_{V_{1}} \boldsymbol{\sigma}^{T}(\delta \varepsilon) d V_{1}=\delta \mathbf{u}_{n}^{T} \mathbf{K} \mathbf{u}_{n},
$$

where $V_{1}$ is the volume of the element in the local coordinate system, $\boldsymbol{\sigma}$ is the element stress, $\varepsilon$ is the element strain, $\mathbf{u}_{n}$ is the nodal displacement, and $\mathbf{K}$ is the stiffness matrix of element formulated as follows

$$
\mathbf{K}=\iiint_{V_{1}} \mathbf{B}^{T} \mathbf{D B} d V_{1},
$$

where $\mathbf{B}$ is the transformation matrix and $\mathbf{D}$ is the constitutive matrix.

The integration of formula (6), in the local coordinate system $x y z$, can hardly be solved. However, in the natural coordinate system $\xi \eta \zeta$, in which the origin is located at the center of the cube element, coordinates of element nodes are transformed, and the stiffness matrix $\mathbf{K}$ can be calculated as

$$
\mathbf{K}=\int_{-1}^{1} \int_{-1}^{1} \int_{-1}^{1} \mathbf{B}^{T} \mathbf{D B} \operatorname{det}(\mathbf{J}) d \xi d \eta d \zeta,
$$

where $\mathbf{J}$ is the Jacobian matrix that represents the relationship of partial derivatives between local and natural coordinates.

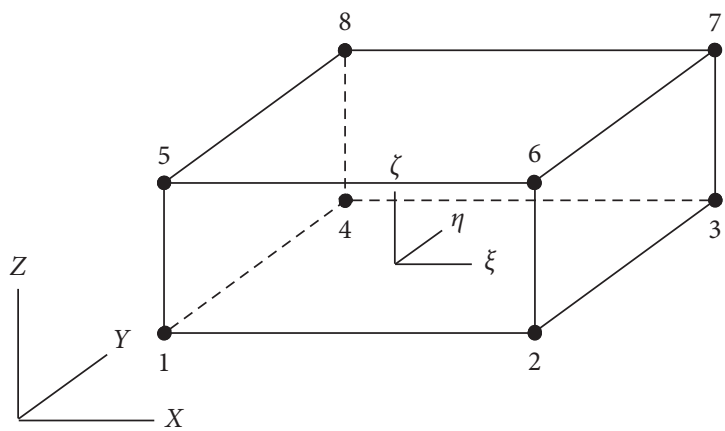

Figure 1: Thin-layer element.

Apparently, in the natural coordinate system, numerical integration is simplified. As shown in Figure 1, the Jacobian matrix $\mathbf{J}$ is derived and simplified as follows:

$$
\mathbf{J}=\left[\begin{array}{lll}
\partial x / \partial \xi & \partial y / \partial \xi & \partial z / \partial \xi \\
\partial x / \partial \eta & \partial y / \partial \eta & \partial z / \partial \eta \\
\partial x / \partial \zeta & \partial y / \partial \zeta & \partial z / \partial \zeta
\end{array}\right]=\left[\begin{array}{ccc}
l / 2 & 0 & 0 \\
& w / 2 & 0 \\
s y m & & t / 2
\end{array}\right] .
$$

Substituting formula (8) into (7) and adopting Gaussian integral method, the stiffness matrix $\mathbf{K}$ can be numerically solved

$$
\begin{aligned}
\mathbf{K}= & \sum_{m=1}^{2} \sum_{n=1}^{2} \sum_{p=1}^{2} \mathbf{B}\left(\xi_{m}, \eta_{n}, \zeta_{p}\right)^{T} \mathbf{D B}\left(\xi_{m}, \eta_{n}, \zeta_{p}\right) \operatorname{det} \\
& \cdot\left(\mathbf{J}\left(\xi_{m}, \eta_{n}, \zeta_{p}\right)\right) w_{\xi_{m}} w_{\eta_{n}} w_{\zeta_{p}},
\end{aligned}
$$

where $w_{\xi_{m}}, w_{\eta_{n}}$, and $w_{\zeta_{p}}$ are weight coefficients of numerical integration.

In the thin-layer element, the thickness of element $t$ is very small and close to zero. According to Desai et al., a constitutive relationship with zero Poisson's ratio results in the decoupling between the normal and tangential behavior of the thin-layer element and in-plane strains and stresses become ignorable. That is, in the coordinate system $x y z$, if the $z$-direction is defined as the normal of the contact region, tangential strain components $\varepsilon_{x}, \varepsilon_{y}$, and $\gamma_{x y}$ and tangential stress components $\sigma_{x}, \sigma_{y}$, and $\tau_{x y}$ are all approximately equal to zero. As a result, the constitutive relationship of the thin-layer element is simplified as follows:

$$
\left\{\begin{array}{c}
\sigma_{z} \\
\tau_{y z} \\
\tau_{z x}
\end{array}\right\}=\left[\begin{array}{ccc}
E_{z} & & \\
& G_{y z} & \\
& & G_{z x}
\end{array}\right]\left\{\begin{array}{c}
\varepsilon_{z} \\
\gamma_{y z} \\
\gamma_{z x}
\end{array}\right\},
$$

where $\sigma_{z}$ is the normal stress in the $z$-direction and $\tau_{y z}$ and $\tau_{z x}$ are the shear stresses in the $y z$ and $z x$ directions, respectively. $\varepsilon_{z}$ is the normal strain in the $z$ direction, and $\gamma_{y z}$ and $\gamma_{z x}$ are the shear strains in the $y z$ and $z x$ directions, respectively. $E_{z}$ is the normal stiffness, and $G_{y z}$ and $G_{z x}$ are tangential stiffnesses.

Equation (10) is derived on the basis of linear elastic constitutive model. In order to describe the energy dissipation in the joint interface, damping coefficient should be introduced in the modeling phase. However, unlike the modeling of stiffness and mass, there is still a lack of suitable 
damping model for the joint interface [35]. In the engineering practice, constant hysteretic model, which assumes frequency- and amplitude-independent damping properties, is often used in the modeling of joint interface [36]. This model is optional in some commercial finite element codes. While the damping is introduced into the model, a complex stiffness matrix of thin-layer element that contains normal and tangential stiffness is obtained.

The performances of thin-layer element are dependent on the virtual material properties and element thickness. To determine a reasonable thickness of thin-layer element, the max aspect ratio is employed defining as follows:

$$
\operatorname{MAR}=\frac{\max (l, w)}{t}
$$

where $l$ is the length, $w$ is the width, and $t$ is the thickness of thin-layer element, respectively.

However, up to now, no standard is available for selecting the maximum aspect ratio of thin-layer elements. Desai et al. suggested a value less than 100, while Pande and Sharma consider that a value larger than 1000 will eliminate numerical errors significantly $[37,38]$. All these suggestions have their own special conditions. In a finite element analysis, the maximum aspect ratio should be selected according to the special condition and the software used [4].

In general, structures excited by small loading are considered to work in the closed state which means that there is no slippage between components of the overall structures, and the frictional force is large enough to contend with the applied forces. As a result, behaviors of mechanical joints are perceived as linear or can be linearized.

\section{Experiments and Application}

In assembled structures, uncertainties are inevitable in both substructures and bolt joints. As a typical type of assembled structure, bolt lapped plate shears, many common issues of assembled structures are widely used in various engineering fields. For the convenience and without loss of generality, in this paper, modeling and hierarchical stochastic updating of a bolt lapped plate are investigated.

3.1. Model Description. The bolt lapped plate is composed of two plates that are assembled together by two bolts, as shown in Figure 2. The width and thickness of the plates are both $120 \mathrm{~mm}$ and $2.76 \mathrm{~mm}$, and their lengths are $450 \mathrm{~mm}$ and $550 \mathrm{~mm}$, respectively.

3.2. Modeling of Bolt Lapped Structure. In order to separate the uncertainties and to make the modeling convenient, the assembled bolt lapped plate is divided into two substructures, named as plate 1 , plate 2 , and two bolt joints. The two plates are molded utilizing isoparametric hexahedral elements, and the bolt joints are modeled by thin-layer elements. Thin-layer elements are connected with elements of plates by node superposition in the contact region. A lumped point element is created in the location of sensor to consider the weight of sensor and its base. The overall FEM of bolt lapped plate is shown as Figure 3.

The performances of structures are determined by material and connection properties. In the bolt lapped structure, isotropic material properties with nominal values of steel are assigned to substructures and orthogonal anisotropy material properties with virtual parameters are assigned to thin-layer elements. As shown in Figure 2, the length of the contact zone is $40 \mathrm{~mm}$ and the diameter of bolts is $10 \mathrm{~mm}$. The stiffness and damping in the contact zone differ from those in the rest zone of the plates. In order to obtain precise models of substructures, as shown in Figure 3, material properties of elements in the contact zone and those in the rest region on the corresponding plates are defined separately. In addition, the acting force of bolt connection converges in the area close to the bolt and much smaller in the rest area of the contact region. Hence, thin-layer elements in the FEM are divided into three parts, two of them include elements in the range of two radiuses of each bolts, denoted as Bolt 1 and Bolt 2, and another part contains the rest thin-layer elements in the contact region, denoted as Contact. To account for the difference of the connection states, all of the three parts are assigned with distinct virtual material properties. The initial material properties of substructures and thin-layer elements are listed in Tables 2 and 3 , respectively.

Apparently, inherent attributes and structural dynamic responses calculated by the initial FEM built based on nominal values or analyst's experience may differ from those of real structures, more or less. For the further study based on FEM, it is necessary to update variable parameters in the model to narrow the gap between simulations and experimental tests.

3.3. Modal Tests and Data Analysis. A hierarchical test scheme is adopted in the modal tests; that is, substructures are tested firstly and then the overall plates are tested. The single-input single-output (SISO) scheme is adopted during the test process. The test plane is designed according to the Tutorial Guideline VDI 3830: Damping of Materials and Members [39]. All test specimens are hanged horizontally by two rubber ropes on a rigid bracket to simulate the free-free boundary condition. Locations of accelerometers are selected elaborately to ensure that sufficient orders of modes can be identified from tests. Moreover, uncertainties of modal characteristics are unavoidable in substructures and assembled structures. In order to consider the uncertainties of modal characteristics, modal tests of specimens with the same sizes are conducted, and accelerometers are kept at their locations without movement during the test to eliminate the influences of sensors.

In the first stage, modal tests of substructures are conducted. Uncertainties of substructures are introduced by the variance of specimens with the same nominal sizes and material properties, but they are always taken from different production batches. In this paper, ten specimens of each kind of substructures are tested. For the case to eliminate the system error, every specimen is tested for three times in the 


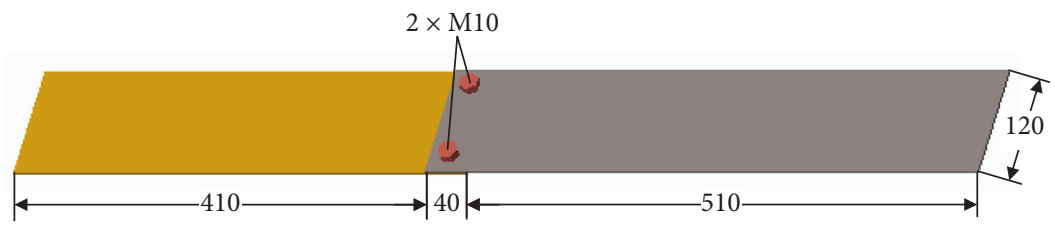

Figure 2: Bolt lapped plate.

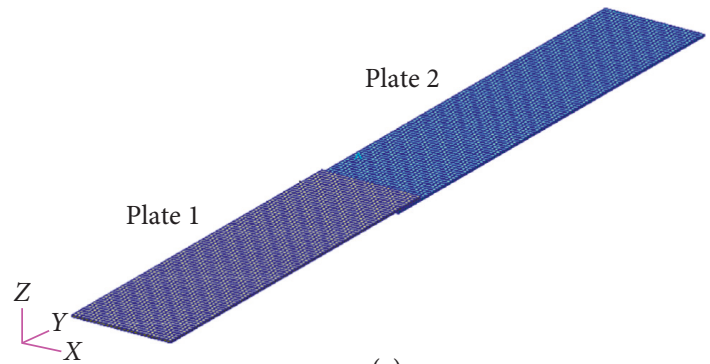

(a)

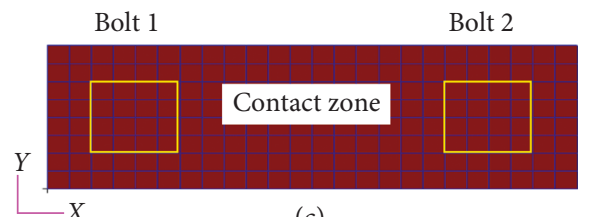

(c)
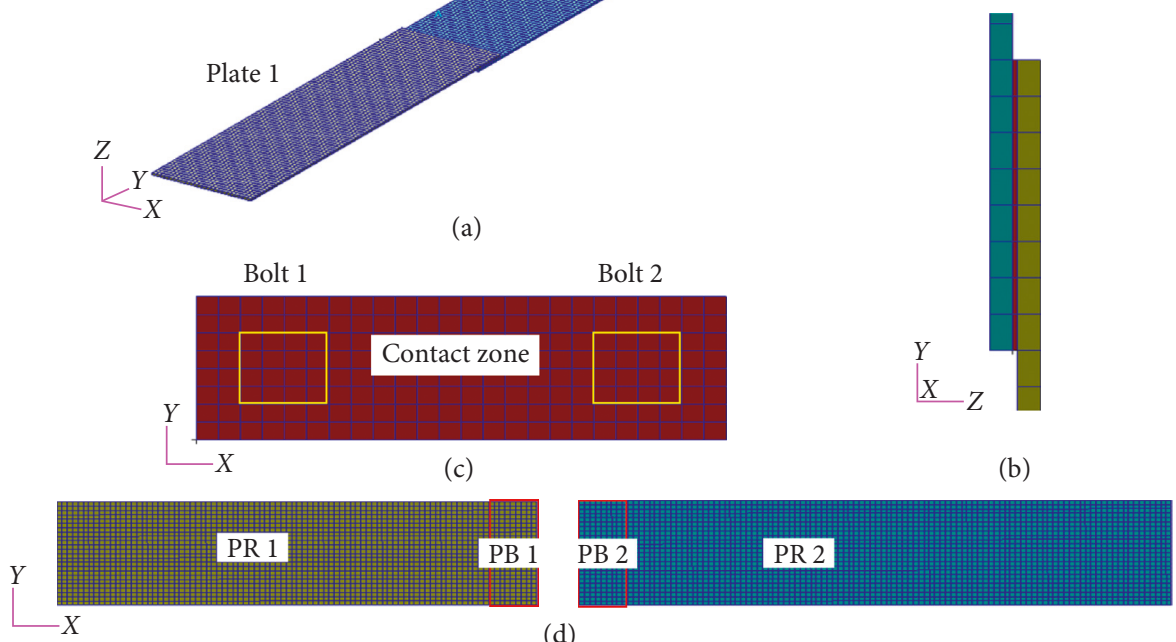

(d)

Figure 3: (a) FEM of the overall bolt lapped structure. (b) Partial enlarged detail of contact region. (c) Locations of bolts. (d) Partition of material properties on substructures.

TABLE 2: Material properties of plates.

\begin{tabular}{lcccc}
\hline \multirow{2}{*}{ Parameters } & \multicolumn{2}{c}{ Plate 1 } & \multicolumn{2}{c}{ Plate 2 } \\
& PR 1 & PB 1 & PR 2 & PB 2 \\
\hline Elastic modulus $(\mathrm{GPa})$ & 206 & 206 & 206 & 206 \\
Poisson ratio & 0.30 & 0.30 & 0.30 & 0.30 \\
Density $\left(\mathrm{kg} \cdot \mathrm{m}^{3}\right)$ & 7750 & 7750 & 7750 & 7750 \\
Damping coefficient & 0.001 & 0.001 & 0.001 & 0.001 \\
\hline
\end{tabular}

same condition, and the results are averaged as the final modal parameters. The experimental modal frequencies, modal damping and their statistics characteristics of substructures, are shown in Tables 4 and 5, respectively.

In the second stage, modal tests of overall structure are conducted. The bolt lapped structure is assembled with plates that are randomly selected from the set of substructures tested in the first stage, and the torques used to connect substructures vary from $10 \mathrm{Nm}$ to $30 \mathrm{Nm}$. In this stage, the bolt lapped structures are assembled for fifty times, and modal tests are conducted on every corresponding specimen. As is same to the substructures, every assembly of bolt lapped structure is tested for three times, and the test results are averaged as the final modal parameters. The test site of the overall structure is shown as Figure 4, and the
TABLE 3: Material properties of thin-layer elements.

\begin{tabular}{lccc}
\hline Parameters & Bolt 1 & Bolt 2 & Contact \\
\hline Normal stiffness, $E_{z}(\mathrm{MPa})$ & 20 & 20 & 10 \\
Tangential stiffness, $G_{y z}(\mathrm{MPa})$ & 10 & 10 & 5 \\
Tangential stiffness, $G_{z x}(\mathrm{MPa})$ & 10 & 10 & 5 \\
Damping coefficient & 0.001 & 0.001 & 0.001 \\
\hline
\end{tabular}

identified modal frequencies and their statistic characteristics are shown in Table 6.

3.4. Surrogate Modeling of Structures. In the model updating process, the iterative analysis of FEM with different groups of model parameters is usually timeconsuming and sometimes even impossible to be completed. Surrogate model is a promising alternative of FEM that represents the relationship between model outputs and design variables. In substructures, the elastic modulus, densities, and damping coefficients are selected as design variables, and the six modal frequencies and damping shown in Tables 4 and 5 are selected as objectives for the construction of RBF models. The elastic modulus and density parameters vary from 0.95 to 1.05 times of the corresponding values shown in Table 2 , and the damping 
TABLE 4: Experimental and updated results of plate 1.

\begin{tabular}{|c|c|c|c|c|c|c|c|c|}
\hline \multirow[t]{2}{*}{ Mode shape } & \multicolumn{2}{|c|}{$\begin{array}{c}\text { Experimental } \\
\text { frequencies }(\mathrm{Hz})\end{array}$} & \multicolumn{2}{|c|}{$\begin{array}{l}\text { Experimental } \\
\text { damping (\%) }\end{array}$} & \multicolumn{2}{|c|}{$\begin{array}{c}\text { Updated frequencies } \\
(\mathrm{Hz})\end{array}$} & \multicolumn{2}{|c|}{$\begin{array}{l}\text { Updated damping } \\
(\%)\end{array}$} \\
\hline & Mean & $\mathrm{SD}$ & Mean & $\mathrm{SD}$ & Mean & $\mathrm{SD}$ & Mean & $\mathrm{SD}$ \\
\hline First bending & 73.46 & 1.057 & 0.0522 & 0.0059 & 73.56 & 1.157 & 0.0500 & 0.0072 \\
\hline First torsion & 166.77 & 2.506 & 0.0473 & 0.0078 & 166.50 & 2.700 & 0.0503 & 0.0083 \\
\hline Second bending & 202.40 & 2.882 & 0.0488 & 0.0092 & 202.88 & 3.293 & 0.0501 & 0.0087 \\
\hline Second torsion & 346.89 & 5.124 & 0.0502 & 0.0095 & 346.77 & 5.414 & 0.0512 & 0.0078 \\
\hline Third bending & 399.03 & 5.726 & 0.0509 & 0.0101 & 400.08 & 6.762 & 0.0503 & 0.0083 \\
\hline Third torsion & 545.87 & 7.135 & 0.0519 & 0.0111 & 546.53 & 8.263 & 0.0522 & 0.0091 \\
\hline
\end{tabular}

TAвLe 5: Experimental and updated results of plate 2.

\begin{tabular}{|c|c|c|c|c|c|c|c|c|}
\hline \multirow[t]{2}{*}{ Mode shape } & \multicolumn{2}{|c|}{$\begin{array}{c}\text { Experimental } \\
\text { frequencies }(\mathrm{Hz})\end{array}$} & \multicolumn{2}{|c|}{$\begin{array}{l}\text { Experimental modal } \\
\text { damping }(\%)\end{array}$} & \multicolumn{2}{|c|}{$\begin{array}{c}\text { Updated frequencies } \\
(\mathrm{Hz})\end{array}$} & \multicolumn{2}{|c|}{$\begin{array}{l}\text { Updated modal } \\
\text { damping }(\%)\end{array}$} \\
\hline & Mean & SD & Mean & SD & Mean & SD & Mean & SD \\
\hline First bending & 48.66 & 0.696 & 0.0498 & 0.0046 & 48.81 & 0.565 & 0.0483 & 0.0034 \\
\hline First torsion & 135.98 & 1.471 & 0.0505 & 0.0050 & 134.44 & 1.599 & 0.0483 & 0.0039 \\
\hline Third bending & 264.21 & 3.486 & 0.0508 & 0.0051 & 263.48 & 3.320 & 0.0478 & 0.0046 \\
\hline Second torsion & 277.26 & 3.127 & 0.0504 & 0.0049 & 276.34 & 3.330 & 0.0490 & 0.0042 \\
\hline Third torsion & 603.39 & 7.247 & 0.0495 & 0.0050 & 603.47 & 8.373 & 0.0498 & 0.0062 \\
\hline Forth torsion & 654.54 & 8.414 & 0.0470 & 0.0063 & 653.97 & 9.132 & 0.0484 & 0.0050 \\
\hline
\end{tabular}

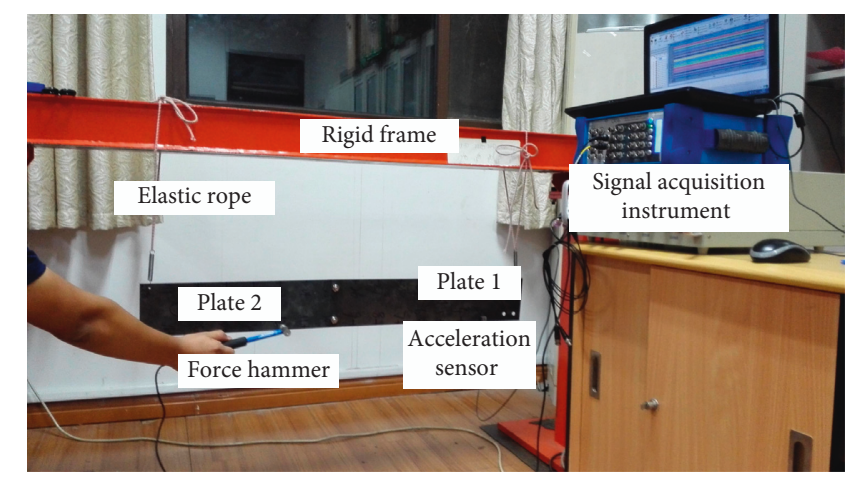

FIgURE 4: Test site of overall plate.

TABLE 6: Experimental and updated results of overall structure.

\begin{tabular}{|c|c|c|c|c|c|c|c|c|}
\hline \multirow[t]{2}{*}{ Mode shape } & \multicolumn{2}{|c|}{$\begin{array}{c}\text { Experimental } \\
\text { frequencies }(\mathrm{Hz})\end{array}$} & \multicolumn{2}{|c|}{$\begin{array}{l}\text { Experimental modal } \\
\text { damping }(\%)\end{array}$} & \multicolumn{2}{|c|}{$\begin{array}{l}\text { Updated frequencies } \\
(\mathrm{Hz})\end{array}$} & \multicolumn{2}{|c|}{$\begin{array}{l}\text { Updated modal } \\
\text { damping (\%) }\end{array}$} \\
\hline & Mean & $\mathrm{SD}$ & Mean & $\mathrm{SD}$ & Mean & SD & Mean & SD \\
\hline First bending & 15.70 & 0.160 & 0.1318 & 0.0194 & 15.74 & 0.178 & 0.1533 & 0.0153 \\
\hline Second bending & 45.35 & 0.457 & 0.3311 & 0.0548 & 45.76 & 0.517 & 0.3299 & 0.0384 \\
\hline Third bending & 80.08 & 1.033 & 0.4418 & 0.1295 & 81.48 & 0.944 & 0.5985 & 0.1004 \\
\hline Forth bending & 141.08 & 1.213 & 0.1660 & 0.0303 & 140.69 & 1.552 & 0.1738 & 0.0267 \\
\hline Second torsion & 148.48 & 1.996 & 0.5461 & 0.0829 & 148.71 & 1.676 & 0.5827 & 0.0708 \\
\hline Fifth bending & 211.38 & 1.742 & 0.0828 & 0.0250 & 213.32 & 2.369 & 0.1012 & 0.0197 \\
\hline
\end{tabular}

parameters vary from 0.1 to 20 times of the corresponding values shown in Table 2 . The assessment criteria, $R^{2}$, are all close to 1 and RMSE varies from $2.26 \times 10^{-6}$ to $2.20 \times 10^{-5}$. The values of assessment criteria indicate that the constructed RBF models of substructures are highly accurate. The RBF model of first bending mode of plate 1 is shown as Figure 5.
In the overall structure, normal stiffness, tangential stiffness, and damping coefficients are selected as design variables, and six modal frequencies and the corresponding modal damping shown in Table 6 are selected as objectives of the construction of surrogate models. The design variables vary from 0.1 to 100 times of the values shown in Table 3. The assessment criteria, $R^{2}$, vary from 0.9904 to 0.9996 , and 


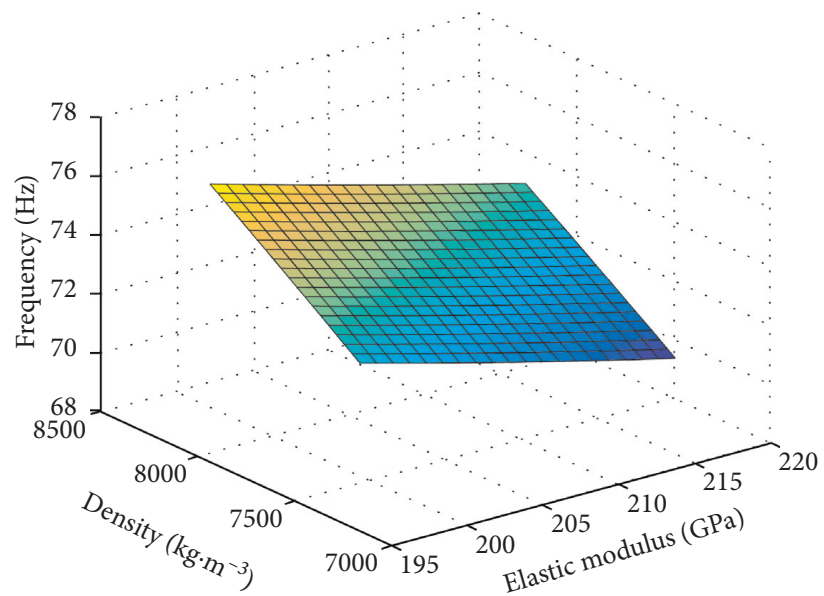

FIgURE 5: RBF model of first bending mode of plate 1.

RMSE varies from $1.53 \times 10^{-4}$ to $1.80 \times 10^{-3}$, which indicates that the constructed RBF models of the bolt lapped plate are also fairly accurate.

3.5. Results of Model Updating. Model updating is completed by revising model parameters to minimize the gap between FEM analysis results and experimental test results. Given the omnipresent uncertainties, all of the model updating tasks are completed under the uncertainty framework hierarchically. Details of updating schemes and results are presented as follows.

In the first stage, elastic modulus, densities, and damping coefficients of substructures are selected as updating parameters. During the process of stochastic model updating, the parameters are assumed to obey the prior multidimensional normal distribution and parameter boundaries are the same as those used in constructing surrogate models. Totally, 10000 sampling points are generated using the DRAM algorithm. Based on the experimental tests and constructed RBF models, updating parameters are calibrated. Statistical characteristic values, that is, mean values (Mean) and standard variances (SD) of updating parameters after updating are shown in Table 7.

After completing the process of stochastic model updating of substructures, a set of 10000 sampling points in the $99 \%$ confidence interval of updating parameters is generated to validate the accuracy of updated results. The statistical characteristics of experimental and updated modal frequencies of plate 1 and plate 2 are listed in Tables 4 and 5, respectively.

As shown in Table 4, the maximum error of modal frequency mean value is $0.26 \%$, and that of standard variance value is $18.09 \%$. The absolute mean error of modal frequency mean values is $0.16 \%$, and that of modal frequency standard variances is $11.84 \%$. In addition, the maximum error of modal damping mean value is $6.34 \%$, and that of standard variance value is $22.03 \%$. The absolute mean error of modal damping mean values is $2.83 \%$, and that of modal frequency standard variances is $14.60 \%$.
Meanwhile, as shown in Table 5, the maximum error of modal frequency mean value is $-1.13 \%$, and that of standard variance value is $18.82 \%$. The absolute mean error of modal frequency mean values is $0.36 \%$, and that of modal frequency standard variances is $10.47 \%$. In addition, the maximum error of modal damping mean value is $-5.91 \%$, and that of standard variance value is $-26.09 \%$. The absolute mean error of modal damping mean values is $3.27 \%$, and that of modal frequency standard variances is $19.47 \%$.

In the second stage, stochastic model updating of the overall structure based on Bayesian inference is conducted. After the work done in the first stage, the statistic characteristics of material properties in the substructures are determined. During the process of updating for the overall structure, updated parameters of substructures are substituted into the model of overall structure and virtual material properties of thin-layer elements are assigned as the updating parameters. As mentioned above, thin-layer elements are divided into three parts in the modeling procedure, that is, Bolt 1, Bolt 2, and Contact region. All of these parts are mutually independent, and they are assigned with individual parameters. During the updating process, one normal stiffness, two tangential stiffness, and damping coefficient of every part thin-layer elements are assigned as updating parameters; that is, totally twelve parameters are allowed to vary until the stable convergence. For the convenience of listing the results of model updating, normal stiffness of Bolt 1, Bolt 2, and Contact region is assigned as $E_{1}, E_{2}$ and $E_{3}$, tangential stiffness is assigned as $G_{y z 1}, G_{z x 1}$, $G_{y z 2}, G_{z x 2}, G_{y z 3}$, and $G_{z x 3}$, and the corresponding damping coefficients are assigned as $\eta_{1}, \eta_{2}$, and $\eta_{3}$, respectively. During the process of stochastic model updating, parameters are assumed to obey the prior multidimensional normal distribution, and variable spaces of updating parameters are the same as those used in constructing surrogate models. The advanced MCMC method DRAM algorithm with 10000 evaluations is adopted to determine the posterior PDFs of updating parameters. Statistic characteristics of updating parameters are listed in Table 8 , and the comparison between experimental and updated results is shown in Table 6. 
TABLE 7: Updated parameters of substructures.

\begin{tabular}{|c|c|c|c|c|c|c|c|c|}
\hline \multirow{2}{*}{ Parameters } & \multicolumn{2}{|c|}{ PR 1} & \multicolumn{2}{|c|}{ PB 1} & \multicolumn{2}{|c|}{ PR 2} & \multicolumn{2}{|c|}{ PB 2} \\
\hline & Mean & $\mathrm{SD}$ & Mean & SD & Mean & SD & Mean & $\mathrm{SD}$ \\
\hline Elastic modulus (GPa) & 203.19 & 8.39 & 181.61 & 15.21 & 201.06 & 5.91 & 178.42 & 28.04 \\
\hline Density $\left(\mathrm{kg} \cdot \mathrm{m}^{-3}\right)$ & 7465.57 & 259.42 & 7344.51 & 220.51 & 7379.71 & 221.26 & 6517.86 & 525.78 \\
\hline Damping coefficient, $\eta_{\mathrm{p}}(\%)$ & 0.0463 & 0.0026 & 0.0830 & 0.0054 & 0.0427 & 0.0034 & 0.0557 & 0.0028 \\
\hline
\end{tabular}

TABLE 8: Updated parameters of overall substructure.

\begin{tabular}{lcc}
\hline Parameter & Mean $(\mathrm{MPa})$ & SD $(\mathrm{MPa})$ \\
\hline Normal stiffness, $E_{1}(\mathrm{MPa})$ & 80.09 & 8.562 \\
Tangential stiffness, $G_{y z 1}(\mathrm{MPa})$ & 39.81 & 4.031 \\
Tangential stiffness, $G_{z x 1}(\mathrm{MPa})$ & 39.97 & 3.879 \\
Damping coefficient, $\eta_{1}(\%)$ & 0.107 \\
Normal stiffness, $E_{2}(\mathrm{MPa})$ & 80.11 \\
Tangential stiffness, $G_{y z 2}(\mathrm{MPa})$ & 39.94 \\
Tangential stiffness, $G_{z x 2}(\mathrm{MPa})$ & 40.12 \\
Damping coefficient, $\eta_{2}(\%)$ & 0.120 \\
Normal stiffness, $E_{3}(\mathrm{MPa})$ & 50.01 \\
Tangential stiffness, $G_{y z 3}(\mathrm{MPa})$ & 25.01 \\
Tangential stiffness, $G_{z x 3}(\mathrm{MPa})$ & 25.07 \\
Damping coefficient, $\eta_{3}(\%)$ & 0.115 \\
\hline
\end{tabular}

As shown in Table 6, the maximum error of modal frequency mean value is $1.75 \%$, and that of standard variance value is $35.99 \%$. The absolute mean error of modal frequency mean value is $0.71 \%$, and that of standard variance value is $18.83 \%$. However, when it comes to damping, the maximum error of modal frequency mean value is $35.47 \%$, and that of standard variance value is $-29.93 \%$. The absolute mean error of modal frequency mean value is $14.29 \%$, and that of standard variance value is $20.20 \%$.

The results shown in Tables 4-6 indicate that favorable agreements between experimental results and updated results of modal frequencies and modal damping are obtained by stochastic model updating based on the Bayesian inference.

Modal frequencies and modal damping calculated by sampling points generated according to the initial parameters and updated parameters, as well as the experimental results are shown in Figure 6.

As shown in Figure 6, the modal frequencies and damping between the results calculated by the initial parameters and the updated results are obvious. However, the updated results are closer to the experimental ones and the differences.

The purpose of model updating is to obtain a FEM with a high precision which can predict some structural characteristics or responses that are not used in the updating process. Modal frequencies and modal damping of third torsion and sixth bending are used to validate the prediction capability of the updated FEM. Mean value and standard variance of modal frequencies and modal damping used in validation are listed in Table 9.

As shown in Table 9, the statistics characteristics of modal frequency used to validate the precision of FEM are very close to the experimental ones. The maximum error of modal frequency mean value is $1.01 \%$, and that of standard variance value is $-10.71 \%$. The absolute mean error of modal frequency mean value is $0.83 \%$, and that of standard variance value is $5.85 \%$. Meanwhile, the maximum error of modal damping mean value is $-22.77 \%$, and that of standard variance value is $-26.91 \%$. The absolute mean error of modal damping mean value is $16.38 \%$, and that of standard variance value is $21.29 \%$.

Moreover, a rough estimation of the time consumed in stochastic model updating of the overall bolt lapped structure with 10000 iterations based on FEM directly is about 4.6 days that means that it has a huge amount of computation. However, time consumed in the process of stochastic modal updating with the same times of iterations based on RBF models of the overall bolt lapped structure is about 4 minutes. The comparison of consumed time used in the model updating indicates that model updating based on surrogate models is a desirable way to save the computational cost.

\section{Conclusion}

In this paper, the issue of hierarchical finite element modeling and stochastic model updating of a bolt lapped plate is presented. The whole procedure is implemented in a progressive manner. In the part of modeling, isoparametric hexahedron elements and thin-layer elements with virtual material properties are adopted to represent substructures and bolt joint interfaces, respectively. Thin-layer elements make it possible to assign different material properties that represent the variance of interface stiffness and damping. In the part of stochastic model updating, material properties of substructures are calibrated firstly, and then virtual material properties that represent the interface stiffness and damping 

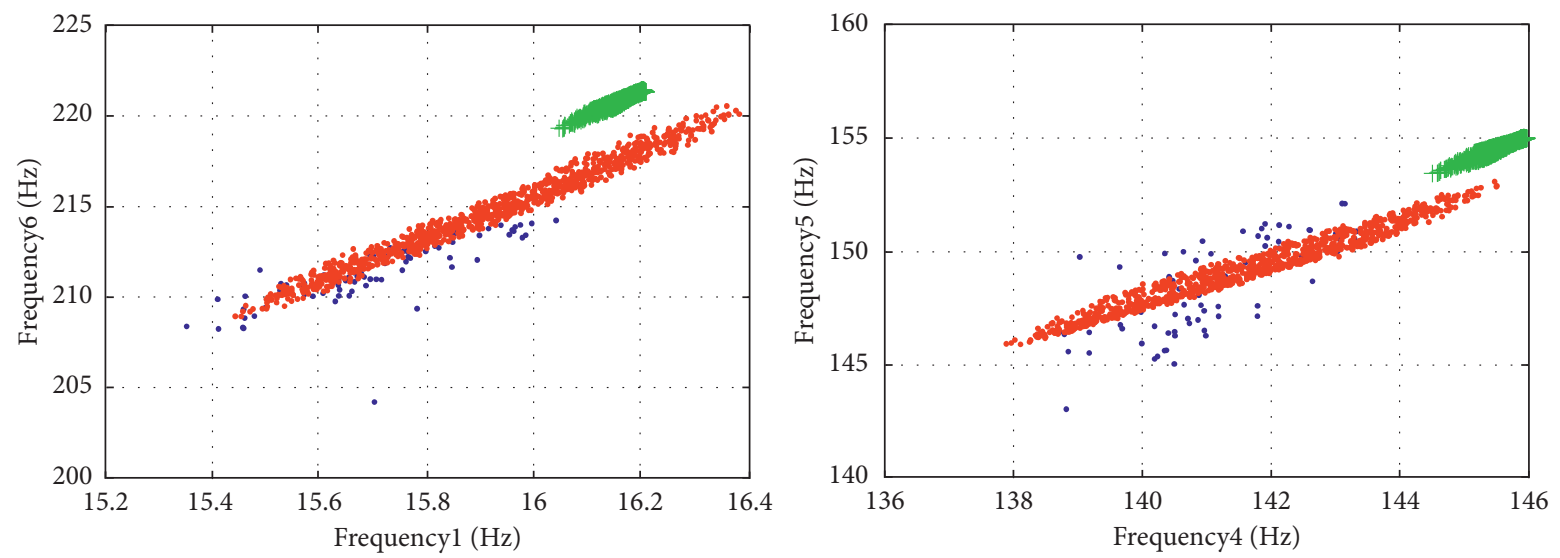

- Experimental results

+ Initial results

- Updated results

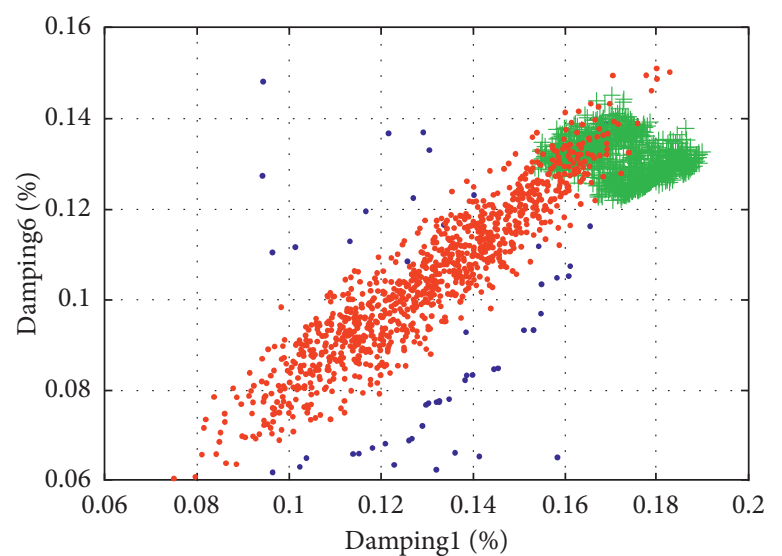

- Experimental results

+ Initial results

- Updated results

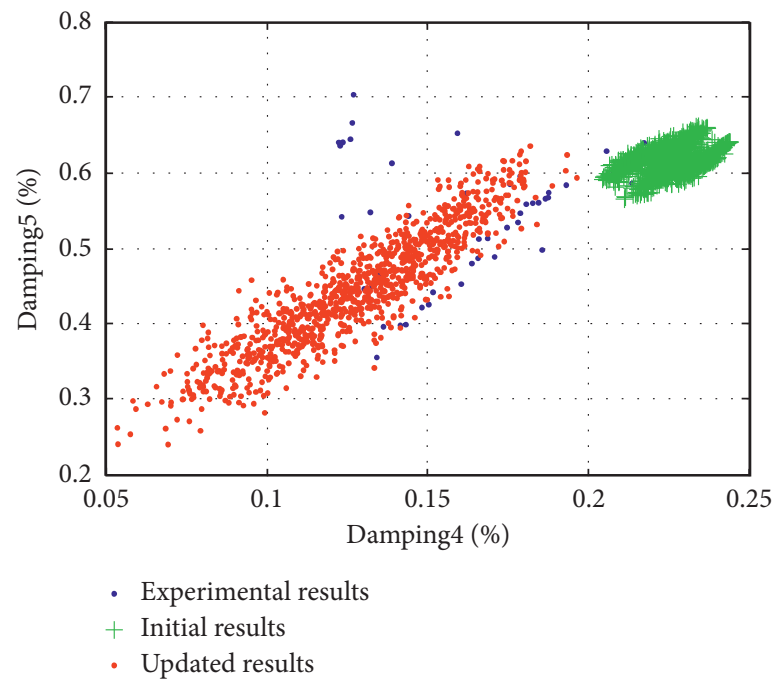

- Experimental results

+ Initial results

- Updated results

- Updated results

FIGURE 6: Comparison of modal frequencies and modal damping.

TABLE 9: Results of validating modal frequencies.

\begin{tabular}{|c|c|c|c|c|c|c|c|c|}
\hline \multirow[t]{2}{*}{ Mode shape } & \multicolumn{2}{|c|}{$\begin{array}{c}\text { Experimental } \\
\text { frequencies }(\mathrm{Hz})\end{array}$} & \multicolumn{2}{|c|}{$\begin{array}{l}\text { Experimental modal } \\
\text { damping (\%) }\end{array}$} & \multicolumn{2}{|c|}{$\begin{array}{c}\text { Updated frequencies } \\
(\mathrm{Hz})\end{array}$} & \multicolumn{2}{|c|}{$\begin{array}{l}\text { Updated modal } \\
\text { damping (\%) }\end{array}$} \\
\hline & Mean & $\mathrm{SD}$ & Mean & $\mathrm{SD}$ & Mean & $\mathrm{SD}$ & Mean & SD \\
\hline Third torsion & 241.07 & 4.303 & 0.2923 & 0.0843 & 242.64 & 3.842 & 0.2631 & 0.0711 \\
\hline Sixth bending & 292.32 & 3.551 & 0.0896 & 0.0457 & 295.27 & 3.516 & 0.0692 & 0.0334 \\
\hline
\end{tabular}

are calibrated. However, stochastic model updating based on the Bayesian inference requires a large amount of sampling points, and the direct model analysis at these sampling points is time-consuming. To solve this problem, surrogate models named as RBF models and an advanced MCMC algorithm named as DRAM algorithm are employed in this paper. The modal frequencies and modal damping calculated from the updated model show a great agreement with the corresponding experimental results. As a consequence, the strategy combining surrogate models and Bayesian inference in the stochastic model updating is a commendable way to improve the precision of FEM and enhancing computational efficiency in engineering practices.

\section{Data Availability}

The data used to support the findings of this study are available from the corresponding author upon request.

\section{Conflicts of Interest}

The authors declare that they have no conflicts of interest.

\section{Acknowledgments}

This work was financially supported by the National Science Foundation of China (Grant nos. 51505398 and U1530122) 
and the Aeronautical Science Foundation of China (20150968003).

\section{References}

[1] W. L. Oberkampf and C. J. Roy, Verification and Validation in Scientific Computing, Cambridge University Press, Cambridge, UK, 2010.

[2] S. Bograd, P. Reuss, A. Schmidt, L. Gaul, and M. Mayer, "Modeling the dynamics of mechanical joints," Mechanical Systems and Signal Processing, vol. 25, no. 8, pp. 2801-2826, 2011.

[3] D. J. Segalman, D. L. Gregory, and M. J. Starr, "Handbook on dynamics of jointed structures," Technical Report SAND2009-4164, Sandia National Laboratories, Albuquerque, NM, USA, 2009.

[4] M. H. Mayer and L. Gaul, "Segment-to-segment contact elements for modelling joint interfaces in finite element analysis," Mechanical Systems and Signal Processing, vol. 21, no. 2, pp. 724-734, 2007.

[5] Y. Luan, Z. Q. Guan, G. D. Cheng, and S. Liu, “A simplified nonlinear dynamic model for the analysis of pipe structures with bolted flange joints," Journal of Sound and Vibration, vol. 331, no. 2, pp. 325-344, 2012.

[6] Y. Zhao, C. Yang, L. Cai, W. Shi, and Y. Hong, "Stiffness and damping model of bolted joints with uneven surface contact pressure distribution," Journal of Mechanical Engineering, vol. 62, no. 11, pp. 665-677, 2016.

[7] Y. Li, Z. Hao, J. Feng, and D. Zhang, "Investigation into discretization methods of six-parameter Iwan model," Mechanical Systems and Signal Processing, vol. 85, pp. 98-110, 2017.

[8] Z. Wang, C. W. Fei, and J. J. Wang, "Equivalent simulation of mechanical characteristics for parametric modeling of bolted joint structures," Advances in Mechanical Engineering, vol. 9, no. 6, pp. 1-12, 2017.

[9] J. Xiang, S. Zhao, D. Li, and Y. Wu, “An improved spring method for calculating the load distribution in multi-bolt composite joints," Composites Part B: Engineering, vol. 117, pp. 1-8, 2017.

[10] J. E. Mottershead and M. I. Friswell, "Model updating in structural dynamics: a Survey," Journal of Sound and Vibration, vol. 167, no. 2, pp. 347-375, 1993.

[11] M. I. Friswell and J. E. Mottershead, Finite Element Model Updating in Structural Dynamics, Springer, Dordrecht, Netherlands, 1995.

[12] H. Ahmadian, J. E. Mottershead, S. James, M. I. Friswell, and C. A. Reecec, "Modeling and updating of large surface-tosurface joints in the AWE-MACE structure," Mechanical Systems and Signal Processing, vol. 20, no. 4, pp. 868-880, 2006.

[13] X. Zhai, C. W. Fei, J. J. Wang, and X. Y. Yao, "Parametric modeling and updating for bolted joints of aeroengine casings," Journal of Mechanical Engineering Science, vol. 230, no. 16, pp. 2940-2951, 2016.

[14] J. Cunha, E. Foltete, and N. Bouhaddi, "Evaluation of stiffness of semi-rigid joints in pultruded profiles from dynamic and static data by using model updating technique," Engineering Structures, vol. 30, no. 4, pp. 1024-1036, 2008.

[15] F. Adel, S. Shokrollahi, M. J. Omidi, and H. Ahmadian, "A model updating method for hybrid composite/aluminum bolted joints using modal test data," Journal of Sound and Vibration, vol. 396, pp. 172-185, 2017.
[16] Y. Xiong, W. Chen, K. L. Tsui, and D. W. Apley, “A better understanding of model updating strategies in validating engineering models," Computer Methods in Applied Mechanics and Engineering, vol. 198, no. 15-16, pp. 1327-1337, 2009.

[17] R. A. Ibrahima and C. L. Pettit, "Uncertainties and dynamic problems of bolted joints and other fasteners," Journal of Sound and Vibration, vol. 279, no. 3-5, pp. 857-936, 2005.

[18] H. H. Khodaparast, J. E. Mottershead, and M. I. Friswell, "Perturbation methods for the estimation of parameter variability in stochastic model updating," Mechanical Systems and Signal Processing, vol. 22, no. 8, pp. 1751-1773, 2008.

[19] D. Jiang, Y. Li, Q. Fei, and S. Wu, "Prediction of uncertain elastic parameters of a braided composite," Composite Structures, vol. 126, pp. 123-131, 2015.

[20] H. H. Khodaparast, J. E. Mottershead, and K. J. Badcock, "Interval model updating with irreducible uncertainty using the kriging predictor," Mechanical Systems and Signal Processing, vol. 25, no. 4, pp. 1204-1226, 2011.

[21] C. Mares, J. E. Mottershead, and M. I. Friswell, "Stochastic model updating: part 1-theory and simulated example," Mechanical Systems and Signal Processing, vol. 20, no. 7, pp. 1674-1695, 2006.

[22] T. Haag, S. C. González, and M. Hanss, "Model validation and selection based on inverse fuzzy arithmetic," Mechanical Systems and Signal Processing, vol. 32, pp. 116-134, 2012.

[23] H. P. Wan and W. X. Ren, "Stochastic model updating utilizing Bayesian approach and Gaussian process model," Mechanical Systems and Signal Processing, vol. 70-71, pp. 245-268, 2016.

[24] B. Goller, M. Broggi, A. Calvi, and G. I. Schueller, "A stochastic model updating technique for complex aerospace structures," Finite Elements in Analysis and Design, vol. 47, no. 7, pp. 739-752, 2011.

[25] Q. Rui, H. Ouyang, and H. Y. Wang, "An efficient statistically equivalent reduced method on stochastic model updating," Applied Mathematical Modelling, vol. 37, no. 8, pp. 60796096, 2013.

[26] S. E. Fang, W. X. Ren, and R. Perera, "A stochastic model updating method for parameter variability quantification based on response surface models and Monte Carlo simulation," Mechanical Systems and Signal Processing, vol. 33, pp. 83-96, 2012.

[27] F. Dohnal, B. R. Mace, and N. S. Ferguson, "Joint uncertainty propagation in linear structural dynamics using stochastic reduced basis methods," AIAA Journal, vol. 47, no. 4, pp. 961-969, 2009.

[28] T. Yin, Q. H. Jiang, and K. V. Yuen, "Vibration-based damage detection for structural connections using incomplete modal data by Bayesian approach and model reduction technique," Engineering Structure, vol. 132, pp. 260-277, 2017.

[29] H. Haario, M. Laine, A. Mira, and E. Saksman, "DRAM: efficient adaptive MCMC," Statistics and Computing, vol. 16, no. 4, pp. 339-354, 2006.

[30] Y. Chen, G. Liu, Z. Zhang, and S. Hou, "Integrated design technique for materials and structures of vehicle body under crash safety considerations," Structural and Multidisciplinary Optimization, vol. 56, no. 2, pp. 455-472, 2017.

[31] Y. Q. Ni, X. G. Hua, K. Q. Fan, and J. M. Ko, "Correlating modal properties with temperature using long-term monitoring data and support vector machine technique," Engineering Structures, vol. 27, no. 12, pp. 1762-1773, 2005.

[32] H. P. Wan, Z. Mao, M. D. Todd, and W. X. Ren, "Analytical uncertainty quantification for modal frequencies with 
structural parameter uncertainty using a Gaussian process metamodel," Engineering Structures, vol. 75, pp. 577-589, 2014.

[33] Q. Guo and L. Zhang, "Finite element model updating based on response surface methodology," in Proceedings of 22nd International Modal Analysis Conference, Society for Experimental Mechanics, Dearborn, MI, USA, 2004.

[34] C. S. Desai, M. M. Zaman, J. G. Lightner, and H. J. Siriwardane, "Thin-layer element for interfaces and joints," International Journal for Numerical and Analytical Methods in Geomechanics, vol. 8, no. 1, pp. 19-43, 1984.

[35] A. Schmidt, S. Bograd, and L. Gaul, "Measurement of join patch properties and their integration into finite-element calculations of assembled structures," Shock and Vibration, vol. 19, no. 5, pp. 1125-1133, 2012.

[36] S. Bograd, A. Schmidt, and L. Gaul, "Modeling of damping in bolted structures,” VDI Berichte, vol. 2003, pp. 97-110, 2007.

[37] K. G. Sharma and C. S. Desai, "Analysis and implementation of thin-layer element for interfaces and joints," Journal of Engineering Mechanics, vol. 118, no. 12, pp. 2442-2462, 1992.

[38] G. N. Pande and K. G. Sharma, "On joint/interface elements and associated problems of ill-conditioning," International Journal for Numerical and Analytical Methods in Geomechanics, vol. 3, no. 3, pp. 293-300, 1979.

[39] L. Gaul, "Tutorial guideline VDI 3830: damping of materials and members," in Proceedings of the Conference on Society for Experimental Mechanics Series, Springer, NY, USA, 2011. 


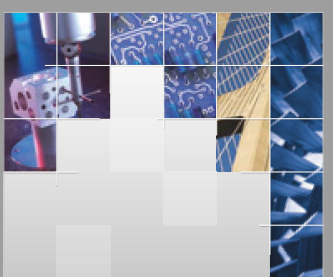

\section{Enfincering}
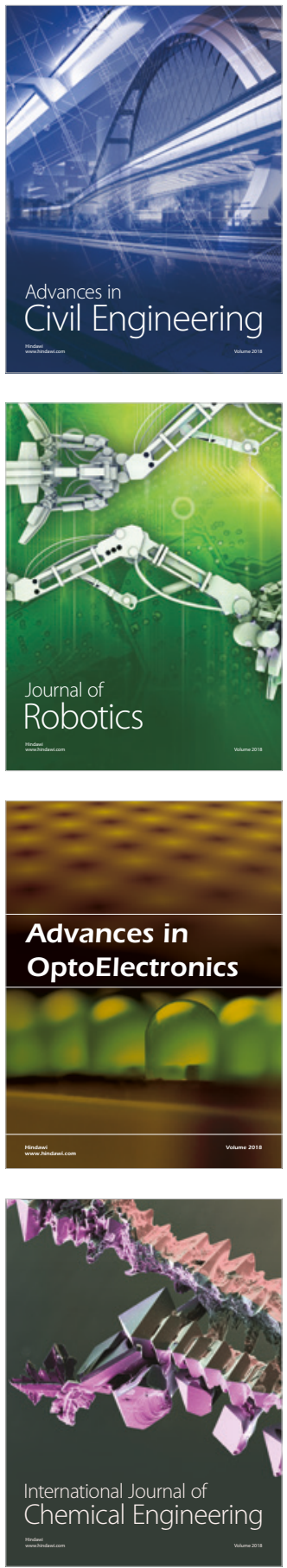

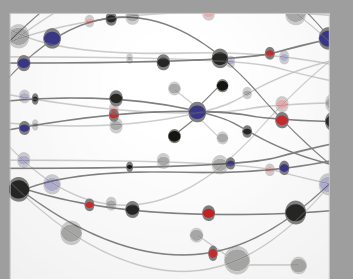

\section{Rotating \\ Machinery}

The Scientific World Journal

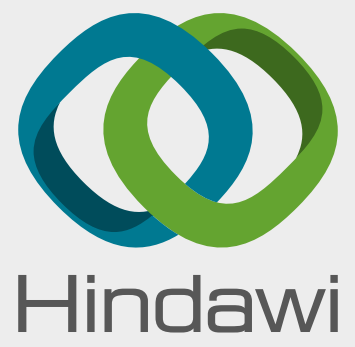

Submit your manuscripts at

www.hindawi.com
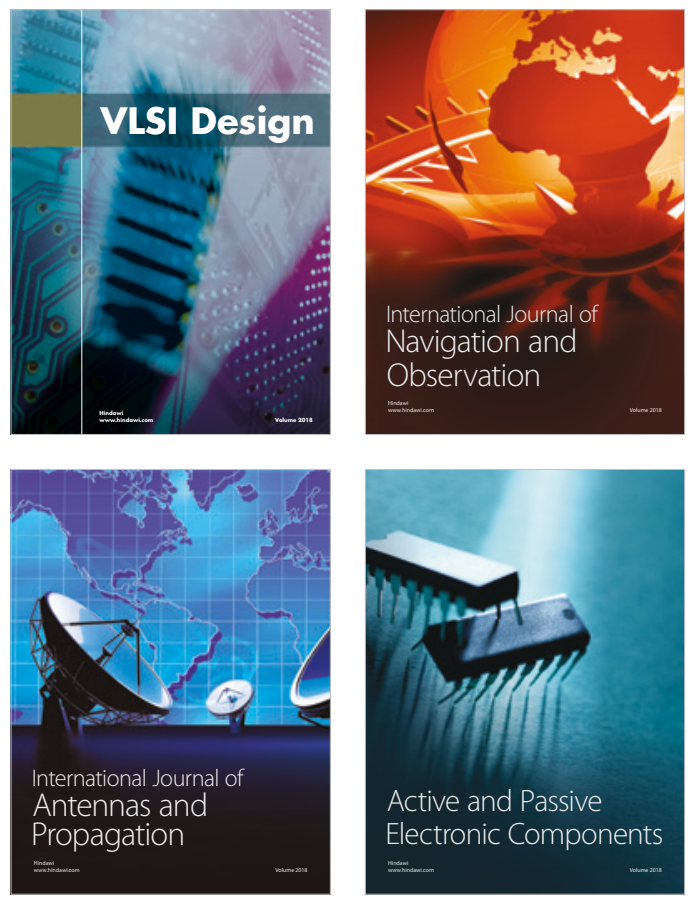
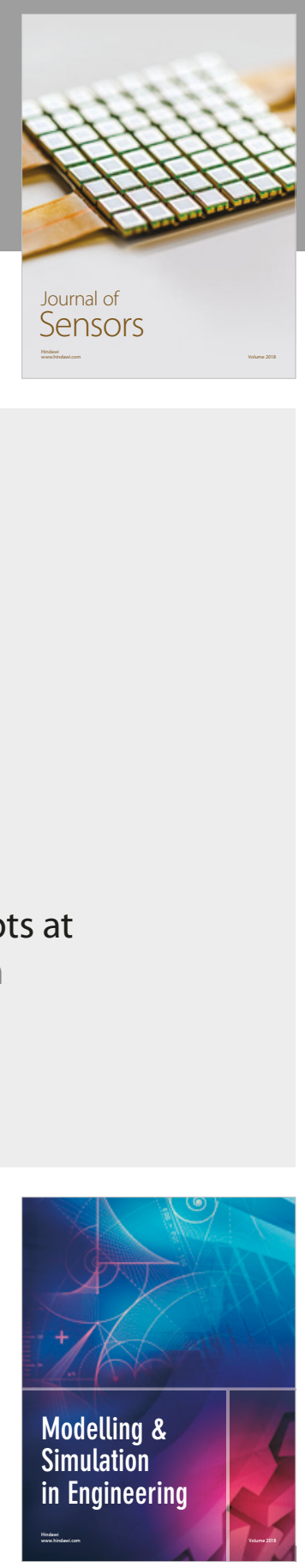

\section{Advances \\ Multimedia}
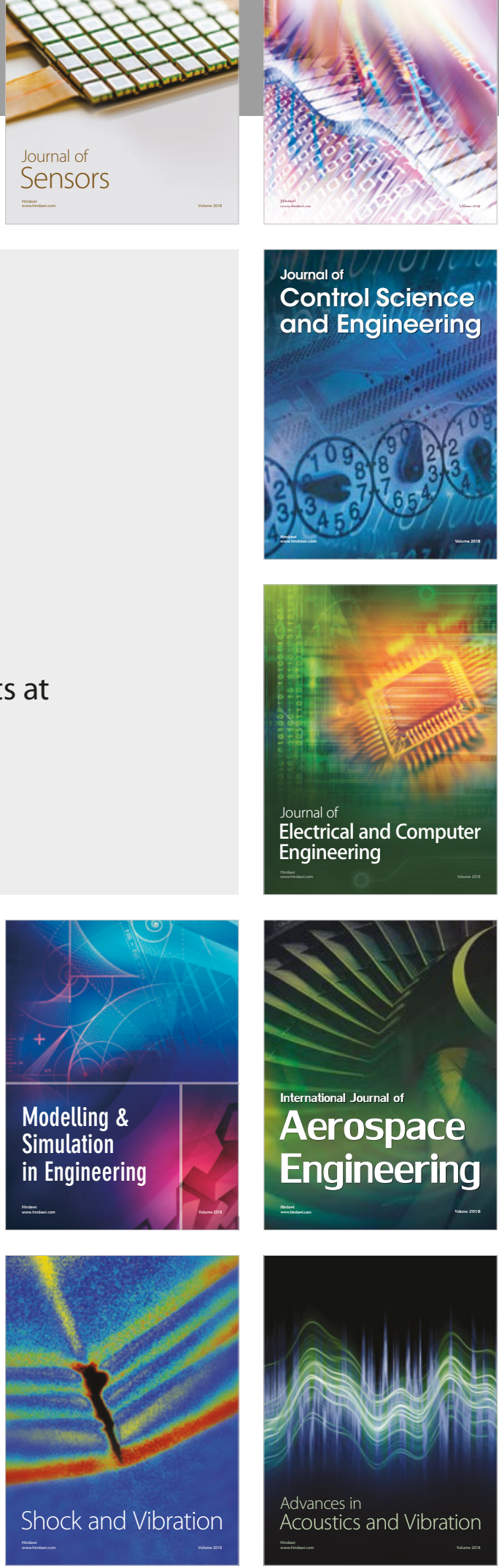